\title{
12 Month Follow Up of Metabolic Measures Following a Randomised Controlled Trial of Treatment of Clozapine Associated Obesity and Diabetes with Exenatide (CODEX)
}

\author{
Siskind, D1,2,3,4, Russell, A2,3, Gamble, C ${ }^{1,4}$, Baker, A4, Cosgrove, P4, Burton, L4, Kisely, $\mathbf{S}^{1,2}$ \\ ${ }^{1}$ Metro South Addiction and Mental Health Service, ${ }^{2}$ The University of Queensland School of Medicine, \\ 3Princess Alexandra Hospital, ${ }^{4}$ Queensland Center for Mental Health Research Brisbane, Qld, Australia
}

\section{Background}

Clozapine is associated with obesity and type 2 diabetes (T2DM). Glucagon-like peptide-1 (GLP-1) receptor agonists such as exenatide can counter clozapine-associated GLP-1 dysregulation in animals, and may be beneficial in people on clozapine. Our randomized, controlled (RCT), open-label, pilot trial of once-weekly extended-release subcutaneous exenatide or treatment as usual (TAU) for 24 weeks $(n=28)$, found 6 of 14 people on exenatide achieved $>5 \%$ weight loss vs 1 of 14 receiving usual care $(P=.029)$. Compared with TAU, participants on exenatide had greater mean weight loss $(-5.29 \mathrm{vs}-1.12 \mathrm{~kg} ; P=.015)$ and body mass index (BMI) reduction $\left(-1.78 \mathrm{vs}-0.39 \mathrm{~kg} / \mathrm{m}^{2} ; \mathrm{P}=.019\right)$, and reduced fasting glucose $(-0.34 \mathrm{vs} 0.39 \mathrm{mmol} / \mathrm{L} ; \mathrm{P}=.036)$ and glycated haemoglobin (HbA1c) levels $(-0.21 \%$ vs $0.03 \% ; \mathrm{P}=.004)$.

\section{Methods}

We followed up CODEX trial participants at 12 months following the end of the trial. We collected information on weight, BMI, waist circumference, blood pressure, fasting glucose, HbA1c, and use of metformin. The primary outcome of interest was change in weight. Change in these parameters from trial baseline to 12 months post endpoint and trial endpoint to 12 months post endpoint was compared between those formerly in the exenatide and TAU arms

\section{Results}

There were no significant differences between baseline and 12-months post endpoint for any of the variables. Data from endpoint to 12-month follow up point showed significantly greater increases among the former exenatide group compared to the former TAU group for weight, BMI, and proportion with $>5 \%$ weight gain. When results for were stratified by whether participants were on metformin six months after the end of the trial ( 5 former TAU and 7 former exenatide were on metformin), the former exenatide group had a greater proportion with $>5 \%$ weight gain between endpoint and 12 months post endpoint irrespective of whether or not they were on metformin.

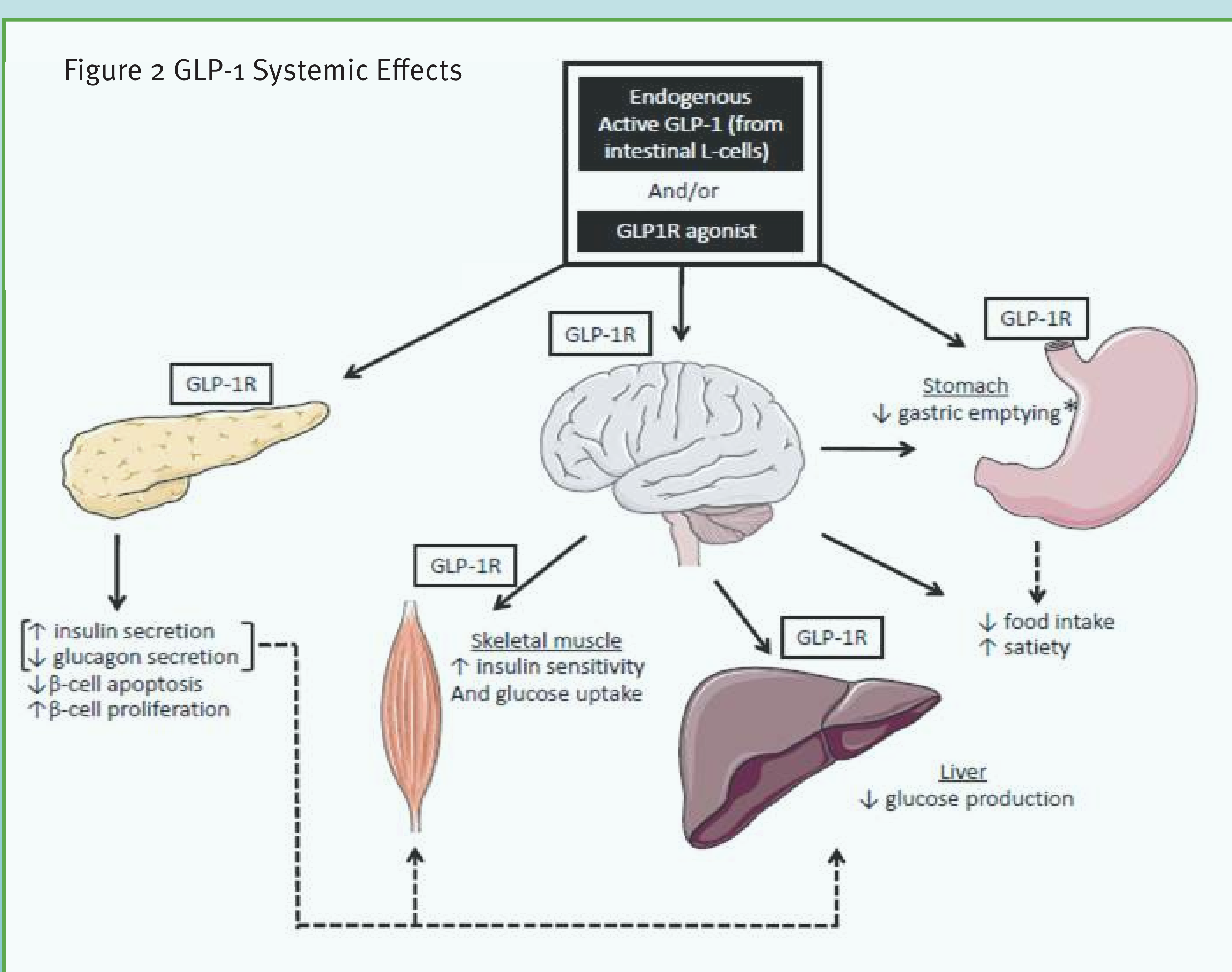

Figure 2: GLP-1 agents regulate satiety behaviour via efferent outflow from the central nervous system (CNS) by both direct activation GLP-1Rs in brain centres involved in feeding (via blood-brain barrier leaks) and indirectly via vagal afferents originating in the intestine. Through the effects of GLP-1R activation in the brain (particularly hypothalamic and brainstem nuclei), GLP-1 and its analogues reduce food intake thereby causing weight loss. Glycaemic control by GLP-1 occurs through the combined contribution of all CNS dependent and independent pathways. Dashed arrows represent indirect effects of GLP-1 not mediated by direct interaction with GLP-1 receptors. Figure adapted from "Heppner KM, Perez-Tilve D. GLP-1 based therapeutics: simultaneously combating T2DM and obesity. Frontiers in neuroscience. 2015;9:92".

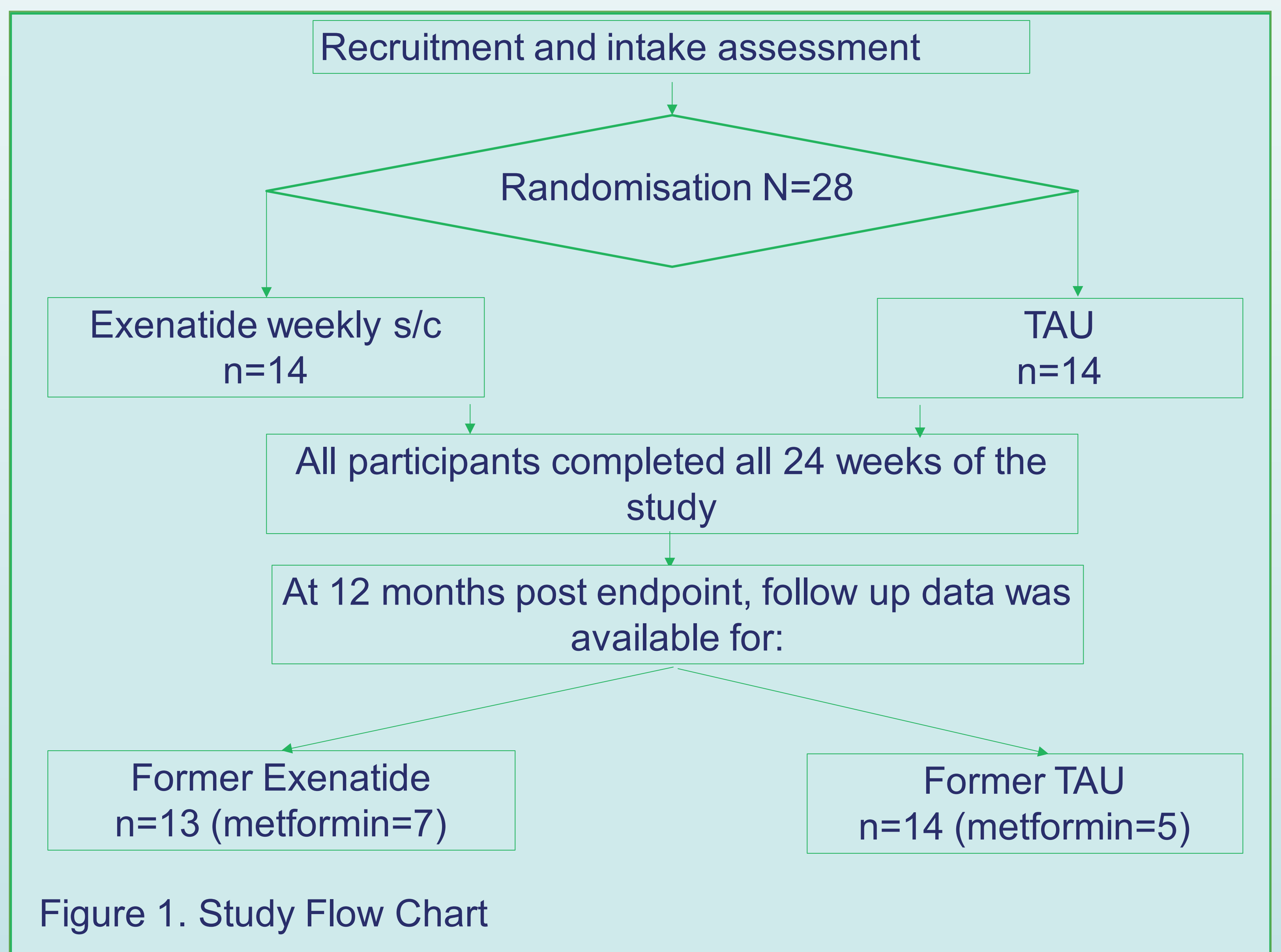

\begin{tabular}{|l|l|l|l|}
\hline Table 1 Change in Variables & Mean Diff & Std. Error & P value \\
\hline Baseline to 12 Months Post & & & \\
\hline Weight & 4.11 & 2.59 & 0.125 \\
\hline BMI & 1.40 & 0.89 & 0.13 \\
\hline Systolic Blood Pressure & 0.70 & 3.49 & 0.843 \\
\hline Diastolic Blood Pressure & -4.02 & 3.21 & 0.224 \\
\hline Waist Circumference & 0.89 & 3.10 & 0.779 \\
\hline Fasting Plasma Glucose & 0.39 & 0.63 & 0.544 \\
\hline HbA1c & 0.68 & 0.25 & 0.022 \\
\hline Triglycerides & 0.02 & 0.46 & 0.970 \\
\hline HDL Cholesterol & 0.03 & 0.08 & 0.711 \\
\hline LDL Cholesterol & -0.02 & 0.39 & 0.968 \\
\hline Total Cholesterol & -0.11 & 0.39 & 0.778 \\
\hline Endpoint to 12 Months Post & & & \\
\hline Weight & 8.28 & 2.03 & $<.0 .001$ \\
\hline BMI & 2.83 & 0.67 & $<0.001$ \\
\hline Systolic Blood Pressure & 2.59 & 3.39 & 0.453 \\
\hline Diastolic Blood Pressure & 2.38 & 3.88 & 0.545 \\
\hline Waist Circumference & 3.04 & 2.81 & 0.296 \\
\hline Fasting Plasma Glucose & 1.24 & 0.68 & 0.084 \\
\hline HbA1C & 0.81 & 0.24 & 0.009 \\
\hline Triglycerides & 0.04 & 0.46 & 0.924 \\
\hline HDL Cholesterol & -0.07 & 0.10 & 0.495 \\
\hline LDL Cholesterol & 0.35 & 0.27 & 0.217 \\
\hline Total Cholesterol & -0.02 & 0.35 & 0.961 \\
\hline
\end{tabular}

\section{Conclusions}

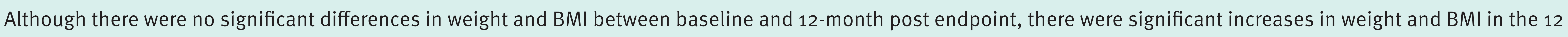

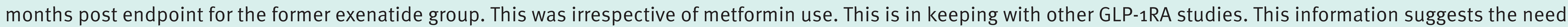
for continued use of exenatide among people on clozapine who have achieved weight loss. 